\title{
Estilos de vida y características sociodemográficas de adolescentes de cinco instituciones educativas
}

\author{
Life style and sociodemographic characteristics of adolescents from five educational \\ institutions
}

\section{Estilos de vida e características demográficas de adolescentes de cindo instituições de ensino}

\author{
Helena Guerrero de Caballero* \\ Astrid Contreras-Orozco**
}

\section{Resumen}

Objetivo: Determinar la relación entre estilos de vida y características sociodemográficas de adolescentes escolarizados en dos subregiones del departamento de Sucre. Colombia. Materiales y Métodos: Estudio descriptivo, transversal y correlacional. La muestra correspondió a 427 estudiantes de básica secundaria de cinco instituciones públicas, con edades comprendidas entre 10 y 15 años, seleccionados por grado escolar, mediante un muestreo sistemático. Se aplicó el Instrumento Fantástico, versión chilena, adaptada en Colombia por Betancurth, Vélez y Jurado (2015) para la caracterización de estilos de vida en la preadolescencia y adolescencia inicial, con niveles óptimos de validez de contenido y fiabilidad (Alpha de Cronbach por criterios): pertinencia $(0,932)$, relevancia $(0,904)$, utilidad $(0,948)$, redacción $(0,846)$ y claridad $(0,800)$. Resultados: La edad promedio fue de 13.1 años, con similitud porcentual por género $(50 \%)$, procedentes de Sincelejo (69.1\%) y Santiago de Tolú (30.9\%), predominando el estilo de vida adecuado en el grupo de 13 a 15 años (32.9 \% y 25.8\%). Los estilos de vida en relación con el género, edad e instituciones educativas por subregión, reportaron diferencias significativas en las dimensiones actividad física y social, sueño y estrés, nutrición, familiares y amigos ( $\mathrm{p}$ $<0.01)$; tipo de personalidad y satisfacción escolar, imagen interior y orden $(\mathrm{p}<0.05)$. Conclusiones: Se encontraron diferencias significativas entre algunas dimensiones de estilos de vida respecto a las variables género, edad, grado escolar e instituciones educativas por subregión de procedencia. Es imperativo realizar estudios de intervención en estilos de vida, para minimizar factores de riesgo en los adolescentes y prevenir enfermedades.

Palabras clave: Condiciones sociales, escolaridad, prevención de enfermedades.

\section{Abstract}

Objective: Determine the relationship between the life styles and the sociodemographic characteristics of school going adolescents in two subregions of the Sucre department, Colombia. Materials and Methods: Descriptive, cross-sectional and correlational study. The sample was made by 427 public middle school students, between

\section{Autor de correspondencia}

* Licenciada en Enfermería. Magíster en Proyectos de Desarrollo Social. Docente Asistente. Programa de Enfermería. Universidad de Sucre. Correo: helena.guerrero@unisucre.edu.co. Orcid: https://orcid. org/0000-0001-5600-1983. Sincelejo, Colombia.

*Enfermera. Magíster en Enfermería énfasis Cuidado para la Salud Cardiovascular. Docente Titular. Programa de Enfermería. Universidad de Sucre. Correo: astrid.contreras@unisucre.edu.co. Orcid: https://orcid. org/0000-0002-6702-8679. Sincelejo, Colombia.
(C) Universidad Francisco de Paula Santander. Este es un artículo bajo la licencia CC BY (https://creativecommons.org/ licenses/by/4.0/) @) (1)

Para citar este artículo/ To reference this article / Para citar este artigo/

Guerrero H, Contreras A.Estilos de vida y características sociodemográficas de adolescentes de cinco instituciones educativas. Rev. cienc. cuidad. 2020; 17(1):85-98. 
ISSN-PRINT

1794-9831

E-ISSN 2322-7028

Vol. 17 No. 1

Ene - Abr 2020

Cúcuta, Colombia

the ages of 10 to 15 , selected through a systematic sample by school grade. The Fantastic Instrument (Chilean version) was applied and adapted to Colombia by Betancourth, Velez and Jurado (2015) for the characterization of preadolescent and adolescent life styles, with optimal levels of content validity and reliability (Cronbach Alpha by criteria): pertinence $(0,932)$, relevance $(0,904)$, utility $(0,948)$, composition $(0,846)$ and clarity $(0,800)$. Results: The average age was 13,1 years, with percentage similarity by gender $(50 \%)$, the students came from Sincelejo $(69,1 \%)$, and Santiago de Tolu $(30,9 \%)$, where the predominant life style was adequate for the age range between 13 and 15 years $(32,9 \%$ and $25,8 \%)$. The life style related to gender, age and educational institutions by subregion, reported significant differences in the dimensions of physical and social activity, sleep and stress, nutrition, family, and friends $(p<0,01)$; type of personality and school satisfaction, inner image and order $(p<0,05)$. Conclusions: Significant differences were found between some life style dimensions with the variables of gender, age, school grade and educational institutions by subregion. It is important for life style intervention studies, to minimize the risk factor for adolescents and prevent diseases.

Key words: Social conditions, schooling, prevention of diseases.

\section{Resumo}

Objetivo: Determinar a relação entre estilos de vida e as características sociais e demográficas de adolescentes escolarizados em duas sub-regiões do estado de Sucre na Colômbia. Materiais e Métodos: Estudo descritivo, transversal e correlacional. A amostra estudada foi de 427 estudantes de ensino médio de cinco escolas públicas, com idades ente 10 e 15 anos, selecionados por nível de escolaridade por amostragem sistemática. Aplicou-se o questionário Fantástico, versão chilena adaptada na Colômbia por Betancurth, Vélez e Jurado (2015) para a caracterização de estilos de vida na pré-adolescência e adolescência inicial, com níveis óptimos de validez de conteúdo e fiabilidade (Alpha de Cronbach por critérios): pertinência $(0,932)$, relevância $(0,904)$, utilidade $(0,948)$, redação $(0,846)$ e claridade $(0,800)$. Resultados: A média da idade foi de13.1 anos, o sexo teve a mesma distribuição (50\%), procedentes da cidade deSincelejo(69.1\%) e Santiago de Tolú (30.9\%), nos quais predominou o estilo de vida adequado no grupo de 13 a 15 anos (32.9\% e $25.8 \%$ ). Os estilos de vida de acordo com o sexo, idade, a escola e a sub-região, reportaram diferenças significativas $(\mathrm{p}<0.01)$; tipo de personalidade, satisfação escolar, imagem interior e ordem $(\mathrm{p}<0.05)$. Conclusões: Encontrou-se diferenças significativas entre algumas dimensões de estilos de vida com as variáveis sexo, idade, nível escolar e escola por sub-região de procedência. É fundamental a realização de estudos de intervenção em estilos de vida, que minimizem os fatores de risco em adolescentes e prevenir doenças.

Palavras-chave: Condições sociais, escolaridade, prevenção de doenças.

\section{Introducción}

La Organización Mundial de la Salud (OMS) define la adolescencia como "el período de crecimiento y desarrollo humano que se produce después de la niñez y antes de la edad adulta, entre los 10 a 19 años" (1). Etapa que se caracteriza por una serie de cambios físicos y psicosociales, como también se consolidan factores de riesgo para la salud, aspectos que conllevan a establecer medidas encaminadas a desarrollar conductas saludables y a controlar los factores modificables en este grupo poblacional $(1,2)$.Cada fase del ciclo de vida del ser humano se constituye en un eslabón fundamental en la adopción de medidas de salud pública, por consiguiente, situaciones que afecten la salud en los primeros años de vida, repercutirán en la adolescencia, a su vez los problemas que ocurran en ésta, afectaran la salud en la adultez(3).
Además, la influencia de lo sociocultural del contexto geográfico es trascendental en el comportamiento de cada individuo o colectivo; de este modo, cada región crea sus propias actuaciones acordes al contexto que le rodea, que deberán ser identificadas como protectores o de riesgo para la salud, por ello, el estilo de vida es un constructo que se usa de manera genérica, en consideración al modo y condiciones de vida (4). Menor et al (5), sostienen que "un estilo de vida saludable es aquel que permite introducir en la manera de vivir pautas de conductas individuales o colectivas, que mejoren la calidad de vida". En este sentido, todas las acciones que fomenten en los adolescentes estilos de vida saludables influirán de manera positiva en su salud y bienestar.

El contexto de éste estudio estuvo circunscrito en dos subregiones del departamento de Sucre (Colom- 
bia), específicamente en los municipios de Santiago de Tolú perteneciente a la subregión del Golfo de Morrosquillo (GM) y en el municipio de Sincelejo, capital del departamento de Sucre, perteneciente a la subregión Sabanas (S). El GM se caracteriza por ser la primera ruta competitiva del departamento, con una oferta turística como un destino diferenciado, con oferta integral de sol y playa como fuente principal de su economía (6).Por su parte, la economía del municipio de Sincelejo se centra en actividades de ganadería, comercio artesanal y agricultura, artesanías en caña flecha y los servicios en menor escala; estas fuentes de ingreso se relacionan con una baja deserción escolar en los adolescentes (7).

Se puede indicar, que los adolescentes como todas las personas, tienen derecho a la vida, a la educación y al acceso a los servicios de salud. Sin embargo, sufren una elevada carga de morbilidad por causas prevenibles, relacionadas principalmente con la violencia, la salud mental, la salud sexual y reproductiva, enfermedades transmisibles y no transmisibles, sedentarismo, consumo de sustancias psicoactivas y conductas autolesivas (8).En los últimos años, cada día fallecen en el mundo más de 3.000 adolescentes por causas prevenibles (4).

Hay que precisar que los estilos de vida influyen en la prevención de las Enfermedades Crónicas No Transmisibles (ECNT) propuestas por la OMS, en donde se señala que en el ámbito mundial existen cuatro enfermedades importantes (cardiovasculares, cáncer, respiratorias crónicas y diabetes) como causa del $82 \%$ de las muertes en todas las edades (9); específicamente, en los adolescentes se relacionan con las cardiovasculares, la leucemia y el asma. En este sentido se reportan como principales factores de riesgo : dieta malsana, inactividad física, consumo de tabaco y consumo nocivo de alcohol; riesgos metabólicos como la obesidad, la hiperglucemia y la diabetes, la hipertensión y la hiperlipidemia $(4,9)$.

En relación a la salud mental de los adolescentes con respecto a los trastornos, estos tienden a asociarse con problemas sanitarios y sociales, como consumo más elevado de alcohol, tabaco y sustancias ilícitas entre otras (10). En los últimos 45 años las tasas de suicidio han aumentado en un $60 \%$ a nivel mundial. El suicidio es una de las tres primeras causas de defunción entre las personas de 15 a 44 años en algunos países y la segunda causa en el grupo de 10 a 24 años (11).
Respecto a la actividad física, estimaciones comparativas indican, que en todo el mundo el $23 \%$ de los adultos y el $81 \%$ de los adolescentes con edades entre 11 y 17 años, no siguen las recomendaciones relativas a la actividad fisica (12). La inactividad física es responsable del 5.5\% de las muertes en el mundo y del 10\% en Europa, que se atribuye en un $10 \%$ a enfermedades cardiovasculares y el $7 \%$ a la diabetes tipo 2 (13).

Con el fin de contribuir a develar los estilos de vida saludables que reduzcan la morbilidad y mortalidad prematura relacionada con las ECNT en la edad adulta, se hace necesario identificar los comportamientos habituales en la adolescencia, dado que muchos jóvenes asumen conductas de riesgo;además, se reporta que entre 82.000 y 99.000 jóvenes empiezan a fumar cada día en el mundo (14).En Colombia, el promedio de edad de inicio en el consumo de alcohol, cigarrillo y sustancias psicoactivas se da en un 13.5 años (15).

Por otro lado, las principales causas de mortalidad registradas en Colombia, entre el 2009 y 2018 ascienden a un total de 49.923 .873 adolescentes entre 12 a 18 años y están asociadas a las ECNT como primera causa, entre las que se citan las afecciones cardiovasculares, el cáncer, la diabetes y los padecimientos pulmonares (16).

Específicamente en el departamento de Sucre, para el grupo de adolescentes no se reportan cifras estadísticas referentes a sus estilos de vida;sin embargo, existe información general, que plantea modificar el comportamiento de los escolares a través de la intervención de los factores conductuales que se relacionen con hábitos desfavorables (17).

En este contexto, la enfermería como disciplina profesional cumple un papel importante dentro del marco de la promoción de la salud, puesto que a través del uso de sus teorías y modelos se han generado acciones para la detección de factores de riesgo, así como para la creación y fortalecimiento de programas que sirvan para el cuidado de las personas, independientemente de su condición de salud (18).Es por eso, que para el presente estudio es de suma importancia identificar hábitos o conductas que afecten el estado de salud de la población de adolescentes escolarizados, que conlleven a proponer en las diversas instituciones educativas, estrategias para el fomento de estilos de vida saludables;de igual forma,se contribuirá a consolidar el trabajo intersectorial en el ámbito de la Estrategia de Atención Primaria en los diversos contextos de la región. 
ISSN-PRINT

1794-9831

E-ISSN 2322-7028

Vol. 17 No. 1

Ene - Abr 2020

Cúcuta, Colombia

\section{Objetivos}

\section{Objetivo General}

Determinar la relación entre los estilos de vida y las características sociodemográficas de los adolescentes de cinco instituciones educativas públicas en dos subregiones del departamento de Sucre(Golfo de Morrosquillo y Sabanas). 2018.

\section{Objetivos Específicos}

- Describir las características sociodemográficas de los adolescentes que cursan básica secundaria de cinco instituciones educativas públicas.

- Describir los estilos de vida de los adolescentes de básica secundaria de cinco instituciones educativas públicas.

- Establecer la relación entre los estilos de vida con las características sociodemográficas de los adolescentes de básica secundaria de cinco instituciones educativas públicas.

\section{Materiales y Métodos}

Es un estudio descriptivo, transversal, correlacional, realizado en el año de 2018 durante seis meses. La población estuvo conformada por 3.780 estudiantes con edades comprendidas entre 10 y 15 años, matriculados en los grados 6,7, 8 y 9 en el período académico de 2018. Se seleccionaron cinco instituciones educativas de carácter público, ubicadas en dos subregiones del departamento de Sucre (Golfo de Morrosquillo y Sabanas). A criterio de las investigadoras se eligieron instituciones educativas con mayor número de estudiantes pertenecientes a cada subregión. En la subregión Golfo de Morrosquillo, se optó por dos instituciones localizadas en el municipio de Santiago de Tolú, con una población de 805 y 355 estudiantes respectivamente; mientras que en Sincelejo se escogieron tres instituciones con 1.415, 796 y 409 estudiantes, respectivamente.

Se escogió una muestra de 427 estudiantes proporcional al número total de cada institución y al grado cursado; se utilizó un nivel de confianza de $95 \%$ y un error de $0,1 \%$. La selección de los estudiantes de cada uno de los grados y grupos de las cinco instituciones educativas se realizó a través de un muestreo sistemático con un intervalo de muestreo de 6. Los criterios de inclusión fueron: la edad,capacidades mentales íntegras y la firma del consentimiento y asentimiento informado.
La información se recolectó a través del Instrumento Fantástico, versión chilena, adaptada y validada en Colombia por Betancurth et al.(19),que sirve para describir el estilo de vida en la pre-adolescencia y adolescencia inicial. La validación se efectuó a través de la técnica Delphi que registró niveles óptimos de validez de contenido y fiabilidad, con un Alpha de Cronbach $>0.80$ en los criterios de: pertinencia, relevancia, utilidad, redacción y claridad. Recolectada la información se procedió a valorar la fiabilidad en la población estudiada y se obtuvo un Alpha de Cronbach de 0.78. Este instrumento está compuesto por 10 dimensiones: 1. Familiares y amigos, 2. Actividad física y social, 3. Nutrición, 4. Toxicidad, 5. Alcohol, 6. Sueño y estrés, 7. Tipo de personalidad y satisfacción escolar, 8. Imagen interior, 9. Control de salud y sexualidad y 10. Orden. El instrumento contempla 3 opciones de respuesta (siempre, algunas veces y nunca) con valor numérico de 0 a 2 para cada categoría, y se califican por medio de una escala tipo Likert, con una calificación de 0 a 120 puntos. A mayor puntaje de la dimensión, la calificación es más positiva hacia la salud. La calificación total se clasifica en los siguientes rangos: de 0 a 46, zona de peligro "estás en zona de peligro (Muy bajo), pero la honestidad es tu real valor"; de 47 a 72 algo bajo "podrías mejorar" (Bajo); de 73 a 84 adecuado "estás bien" (Adecuado); de 85 a 102 "buen trabajo estás en el camino correcto"(Alto) y de 103 a 120 "felicitaciones tienes un estilo de vida fantástico" (Muy alto)(19).

El procesamiento de la información se realizó con el Programa SPSS (Statistical Package for the Social Sciences) versión 24.0. Se efectuaron análisis univariados, bivariados y multivariados para explicar el comportamiento entre las variables del estudio. Se realizaron pruebas de contraste entre las medias de los puntajes de las 10 dimensiones del cuestionario Fantástico, tales como pruebas $t$ sobre muestras independientes para las variables género, rango de edad y lugar de procedencia. También se tuvieron en cuenta análisis de varianza y las correspondientes pruebas para establecer Diferencia Mínima Significativa (DMS) en el contraste entre medias para la variable institución educativa, previa realización de las pruebas de Normalidad y de Homogeneidad de varianza para los puntajes de las dimensiones. De igual manera, se realizaron pruebas $\mathrm{Z}$ de contraste entre las proporciones de respuestas dadas a cada uno de los ítems de las dimensiones, en donde se establecieron diferencias significativas $(* \mathrm{p}<0.05)$ o muy significativas $(* * \mathrm{p}<0.01)$.

El aspecto ético se enmarcó dentro de los principios de respeto a la dignidad humana y confidencialidad 
de la información, acorde a las disposiciones vigentes para la protección de los seres humanos que participan en la investigación, contenidas en la Resolución 8430 de 1993 del Ministerio de Salud de Colombia, clasificando el estudio con riesgo mínimo, según el numeral b del artículo 11(20).Este estudio fue avalado por el Comité de Bioética de la División de Investigación de la Universidad de Sucre. Se explicó a las directivas de las instituciones educativas, padres de familia y estudiantes, los objetivos, metodología y utilidad del estudio;también se solicitó la autorización para su realización y se procedió al diligenciamiento de los Formatos de Consentimiento Informado y de Asentimiento, este último diligenciado por los participantes.

\section{Resultados}

La caracterización sociodemográfica de los 427 participantes del estudio, reportó porcentajes similares por género $(50 \%)$, la edad promedio fue 13.1 años con una desviación estándar de \pm 1.3 años. Los estudiantes proceden del municipio de Sincelejo en un 69.1\%, que pertenece a la subregión Sabanas y capital del departamento de Sucre; y un $30.9 \%$ de Santiago de Tolú, que corresponde a la subregión del Golfo de Morrosquillo. Predominó la religión católica en un 57\%, y los estratos socioeconómicos I y II con un $48.5 \%$ y $44.3 \%$. Con relación al nivel de formación de los padres se encontró, que tanto padres como madres, en gran medida cursaron estudios de básica secundaria completa $(27.9 \%$ y $30.0 \%)$ e incompleta (18.7\% y $21.3 \%)$ respectivamente.

Los resultados generales respecto a las categorías de estilos de vida de los estudiantes y variables sociodemográficas relacionadas con género, edad e instituciones educativas reportaron diferencias estadísticas muy significativas $(\mathrm{p}<0.01)$.El estilo de vida bajo predominó en el género femenino y el estilo adecuado en el masculino, especialmente en los estudiantes con edades comprendidas entre los 13 y 15 años. Por institución educativa, se encontró que la $\mathrm{N}^{\mathrm{o}} 3$ de la subregión Sabanas reportó mayores porcentajes en todas las categorías (Tabla 1).

Tabla 1. Estilo de vida de los estudiantes según género, edad e instituciones educativas de las subregiones Sabanas y Golfo de Morrosquillo del departamento de Sucre, 2018.

\begin{tabular}{|c|c|c|c|c|c|c|c|c|c|}
\hline \multirow{2}{*}{\multicolumn{3}{|c|}{ Variables Sociodemográficas }} & \multicolumn{6}{|c|}{ Estilos de Vida } & \multirow{3}{*}{ Valor $\mathrm{p}$} \\
\hline & & & \multicolumn{2}{|c|}{ Bajo } & \multicolumn{2}{|c|}{ Adecuado } & \multicolumn{2}{|c|}{ Alto } & \\
\hline & $\mathrm{N}^{\circ}$ & $\%$ & $\mathrm{~N}^{\circ}$ & $\%$ & $\mathrm{~N}^{\circ}$ & $\%$ & $\mathrm{~N}^{\circ}$ & $\%$ & \\
\hline \multicolumn{10}{|l|}{ Género } \\
\hline Femenino & 214 & 50,1 & 71 & 62,3 & 90 & 41,5 & 53 & 55,2 & \multirow{2}{*}{$0,001 * *$} \\
\hline Masculino & 213 & 49,9 & 43 & 37,7 & 127 & 58,5 & 43 & 44,8 & \\
\hline \multicolumn{10}{|c|}{ Rangos de Edad (años) } \\
\hline 10 a 12 & 150 & 35,1 & 25 & 21,9 & 86 & 41,5 & 39 & 40,6 & \multirow{2}{*}{$0,003 * *$} \\
\hline 13 a 15 & 277 & 64,9 & 89 & 78,8 & 131 & 58,5 & 57 & 59,4 & \\
\hline \multicolumn{10}{|c|}{ Instituciones Educativas } \\
\hline Institución $1 \mathrm{~S}$ & 91 & 21,3 & 24 & 21,1 & 53 & 24,4 & 14 & 14,6 & \multirow{5}{*}{$0,002 * *$} \\
\hline Institución $2 \mathrm{~S}$ & 45 & 10,5 & 14 & 12,3 & 28 & 12,9 & 3 & 3,1 & \\
\hline Institución $3 \mathrm{~S}$ & 159 & 37,2 & 40 & 35,1 & 81 & 37,3 & 38 & 39,6 & \\
\hline Institución $1 \mathrm{GM}$ & 92 & 21,5 & 19 & 16,7 & 45 & 20,7 & 28 & 29,2 & \\
\hline Institución $2 \mathrm{GM}$ & 40 & 9,4 & 17 & 14,9 & 10 & 4,6 & 13 & 13,5 & \\
\hline
\end{tabular}

Fuente: Resultados de la investigación

S: Subregión Sabanas; GM: Subregión Golfo de Morrosquillo

**Diferencias muy significativas $(\mathrm{p}<0,01)$

Por otra parte, en las subregiones Sabanas y Golfo de Morrosquillo predominó el estilo de vida adecuado en estudiantes con edades que oscilan entre 13 y 15 años, con un $32.9 \%$ y 25.8 . Referente a las variables estilos de vida, grado cursado y subregión de procedencia, se encontró que en la categoría de estilo de vida alto, los estudiantes de los grados séptimo y noveno reportaron el mayor porcentaje en la subregión Golfo de Morrosquillo con un $48.1 \%$ y $36.0 \%$; mientras que en la categoría bajo, el mayor porcentaje correspondió a estudiantes del grado séptimo procedentes de la Sabanas (Figura 1). 
ISSN-PRINT

1794-9831

E-ISSN 2322-7028

Vol. 17 No. 1

Ene - Abr 2020

Cúcuta, Colombia

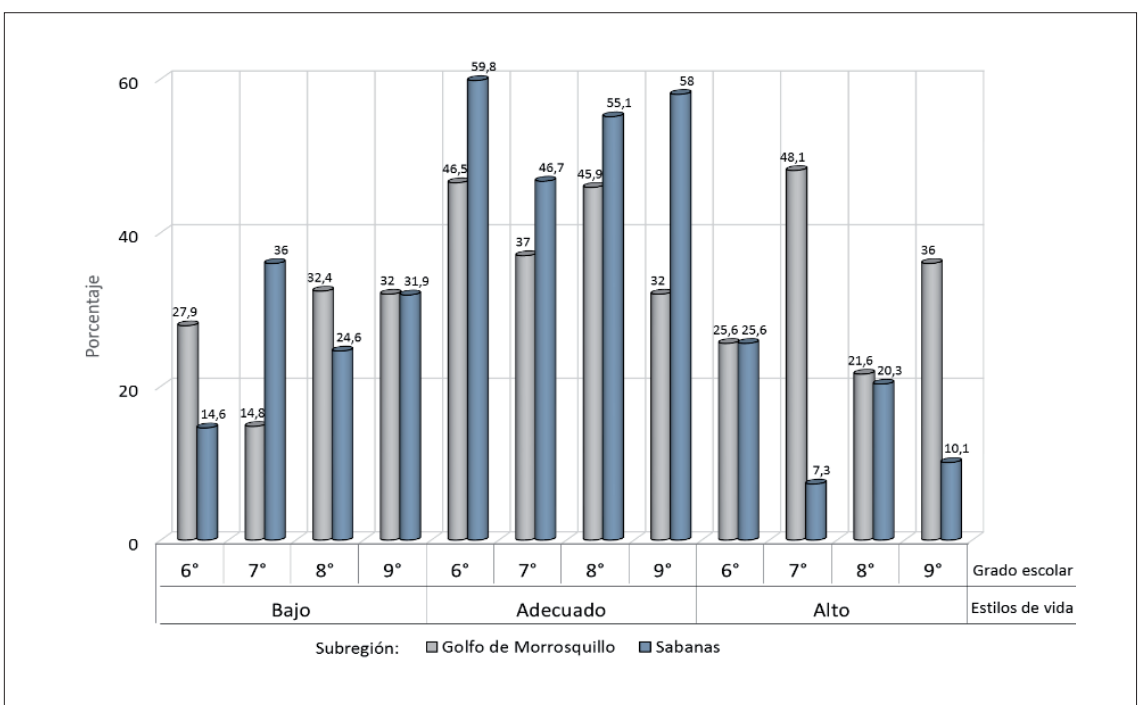

Figura 1. Relación entre estilos de vida, grado cursado y subregión de procedencia.

Fuente: Resultados de la investigación.

Por otra parte, respecto a la relación entre estilos de vida, género y subregión de procedencia,predominó el estilo adecuado en los estudiantes de género masculino de las dos subregiones, en un $60.6 \%$; sin embargo, se observó en esta misma categoría diferencias en el género feme- nino, con un menor porcentaje en las estudiantes procedentes de la subregión Golfo de Morrosquillo. En la categoría estilo de vida bajo, se aprecia que en la subregión Golfo de Morrosquillo el mayor porcentaje corresponde a las estudiantes en un 19.7\%. (Figura 2).

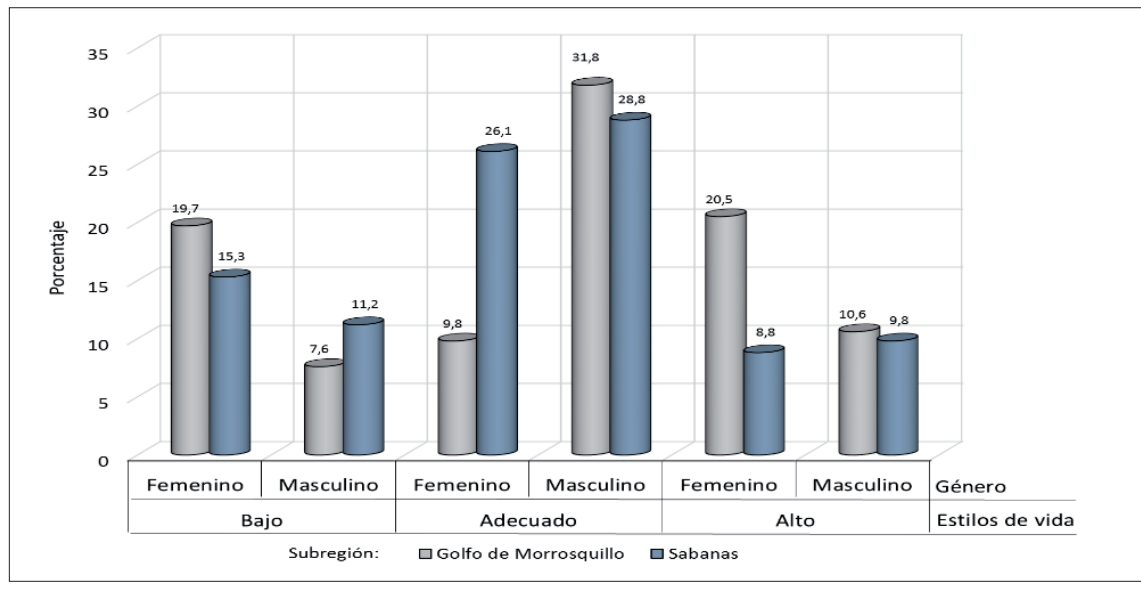

Figura 2. Relación entre estilos de vida, género y subregión de procedencia.

Fuente: Resultados de la investigación.

En cuanto a los estilos de vida entre género e instituciones educativas por subregiones, se encontró que en las dimensiones actividad física y social, sueño y estrés, existían diferencias muy significativas ( $\mathrm{p}$ valor $0.001 \mathrm{y}$ $0.00)$ y en tipo de personalidad y satisfacción escolar, también se observaron diferencias significativas ( $\mathrm{p}$ valor 0.046). En la dimensión actividad física y social se reportaron diferencias por género, con promedios menores
(1.84 y 1.86) en los estudiantes de ambos géneros de la subregión Sabanas con estilo de vida bajo. Respecto a sueño y estrés, la totalidad de los estudiantes de las dos subregiones registraron un estilo de vida alto, a diferencia de la dimensión tipo de personalidad y satisfacción escolar, en la cual los estudiantes de género masculino del Golfo de Morrosquillo, alcanzaron el mayor promedio (5.11) (Tabla 2). 
Tabla 2: Estilos de vida de estudiantes por género e instituciones educativas por subregiones según dimensiones.

\begin{tabular}{|c|c|c|c|c|c|c|}
\hline \multirow{3}{*}{ Género } & \multirow{3}{*}{ Dimensiones } & \multirow{2}{*}{\multicolumn{2}{|c|}{$\begin{array}{l}\text { Instituciones educativas } \\
\text { Golfo de Morrosquillo }\end{array}$}} & \multirow{2}{*}{\multicolumn{2}{|c|}{$\frac{\text { Instituciones educativas }}{\text { Sabanas }}$}} & \multirow{3}{*}{ Valor $\mathrm{p}$} \\
\hline & & & & & & \\
\hline & & $\mathrm{X} \pm \mathrm{S}$ & Estilo de Vida & $\mathrm{X} \pm \mathrm{S}$ & Estilo de Vida & \\
\hline Femenino & \multirow{2}{*}{$\begin{array}{l}\text { Actividad física y } \\
\text { social }\end{array}$} & $2,15 \pm 1,05$ & Adecuado & $1,86 \pm 1,16$ & Bajo & \multirow{2}{*}{$0,001 * *$} \\
\hline Masculino & & $2,15 \pm 1,08$ & Adecuado & $1,84 \pm 1,16$ & Bajo & \\
\hline Femenino & \multirow{2}{*}{ Sueño y estrés } & $4,10 \pm 0,98$ & Alto & $4,07 \pm 1,08$ & Alto & \multirow{2}{*}{$0,000 * *$} \\
\hline Masculino & & $4,16 \pm 0,91$ & Alto & $4,07 \pm 1,07$ & Alto & \\
\hline Femenino & \multirow{2}{*}{$\begin{array}{l}\text { Tipo de personalidad y } \\
\text { satisfacción escolar }\end{array}$} & $4,85 \pm 1,00$ & Bajo & $4,54 \pm 1,07$ & Bajo & \multirow{2}{*}{$0,046^{*}$} \\
\hline Masculino & & $5,11 \pm 0,87$ & Alto & $4,57 \pm 1,08$ & Bajo & \\
\hline
\end{tabular}

ISSN-PRINT

1794-9831

E-ISSN 2322-7028

Vol. 17 No. 1

Ene - Abr 2020

Cúcuta, Colombia

Fuente: Resultados de la investigación

*Diferencias significativas $(\mathrm{p}<0,05)$

**Diferencias muy significativas $(\mathrm{p}<0,01)$

Por otra parte, al evaluar la relación de los estilos de vida entre rangos de edad e instituciones educativas, se encontró que en las dimensiones nutrición, familiares y amigos, se percibían diferencias muy significativas ( $\mathrm{p}$ valor
0.002); mientras que en imagen interior y orden, estas diferencias fueron significativas ( $\mathrm{p}$ valor 0.029 y 0.022 ) (Tabla 3).

Tabla 3. Estilos de vida de estudiantes por rangos de edad e instituciones educativas por subregiones según dimensiones.

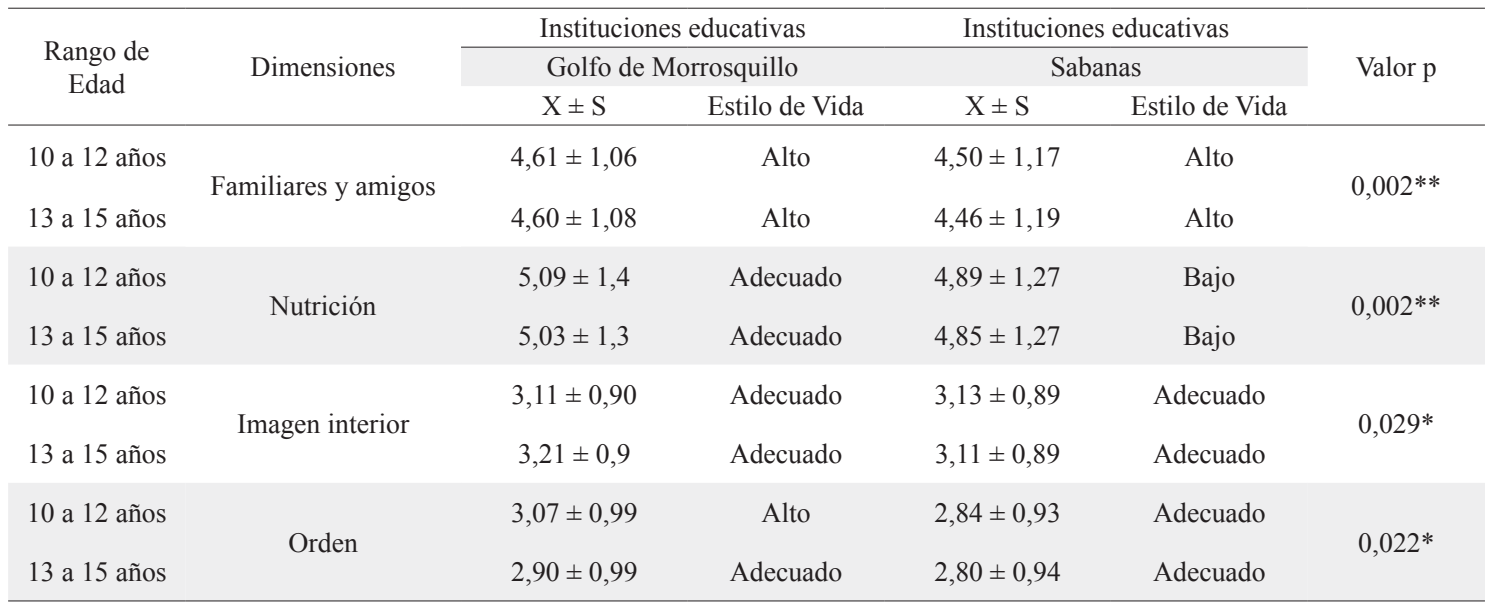

Fuente: Resultados de la investigación

*Diferencias significativas $(\mathrm{p}<0,05)$

**Diferencias muy significativas $(\mathrm{p}<0,01)$

Se destaca que en las dimensiones orden y familiares y amigos, los estudiantes de la subregión Golfo de Morrosquillo, con edades entre 10 a 12 años, obtuvieron promedios mayores (3.07 y 4.61) en la categoría de estilo alto, excepto, en la dimensión imagen interior, que reportó un estilo adecuado para todos los estudiantes, con el promedio mayor (3.21) en los estudiantes del Golfo de Morrosquillo con edades entre 13 a 15 años. Respecto a la nutrición se encontró promedios menores en los estudiantes con estilo de vida bajo de la subregión Sabanas, ubicados en ambos rangos de edad. En las dimensiones toxicidad (hábito de fumar, automedicación y consumo de cafeína), alcohol, control de salud y sexualidad, no se registraron diferencias significativas entre las medias de las puntuaciones ( $p>$ 0.05). En los participantes no se encontraron hábitos nocivos en toxicidad y consumo de alcohol. 
ISSN-PRINT

1794-9831

E-ISSN 2322-7028

Vol. 17 No. 1

Ene - Abr 2020

Cúcuta, Colombia
Cabe señalar, que en la dimensión actividad física y social, en los ítems relacionados a la realización de ejercicios físicos durante los últimos siete días y a la asistencia a grupos deportivos, religiosos o culturales,los hallazgos reportaron diferencias por género $(\mathrm{p}<0.05)$ entre los porcentajes de estudiantes que manifestaron que nunca lo hacen; en la subregión Golfo de Morrosquillo se presentó en un $75.0 \%$ en el género femenino frente al masculino con un $25.0 \%$, y en la subregión Sabanas un $60.6 \%$ en el femenino frente al masculino con un $39.4 \%$. Respecto al tipo de personalidad y satisfacción escolar, se encontraron diferencias $(p<0.05)$ en las dos subregiones en el ítem se siente de buen humor, alegre y activo;en la de Sabanas los estudiantes que expresaron que lo hacen algunas veces, se reportó un $62.8 \%$ en mujeres y el $46.9 \%$ en hombres; mientras que en la subregión Golfo de Morrosquillo el 47.0\% correspondió a mujeres y el $33.3 \%$ a hombres.

Además, en la dimensión sueño y estrés se encontraron diferencias por género $(\mathrm{p}<0.05)$ en dos ítems: 1 . Duermen bien y se sienten descansados al levantarse y 2. Se siente capaz de manejar situaciones estresantes y encontrar fácilmente alternativas de solución. En el primer ítem, predominó la categoría algunas veces, en la subregión Sabanas, con mayor porcentaje en el género femenino que el masculino (60.1\% y 46.9\%); en esta misma categoría, en el Golfo de Morrosquillo se presentó similar comportamiento entre el género femenino y masculino (50 \% y $42.4 \%$ ). En el segundo ítem, los hallazgos mostraron similar comportamiento en ambas subregiones.

En cuanto a la dimensión familiares y amigos, referente a la comunicación y al compartir los sentimientos con otras personas, en el ítem yo recibo cariño, se encontraron diferencias $(\mathrm{p}<0.05)$ en la categoría siempre lo hace, hallándose que en la subregión Sabanas tanto en el grupo etario de 10 a 12 años y en el de 13 a 15 años, se reportaron porcentajes de $65.7 \%$ y $56.1 \%$ respectivamente;mientras que en el Golfo de Morrosquillo los porcentajes fueron de $73.8 \%$ y $61.1 \%$ en los mismos grupos etarios.

Respecto a la dimensión imagen interior, se encontraron diferencias $(p<0.05)$ en el ítem me siento tenso(a) o estresado (a), con predominio de la categoría algunas veces; en este sentido, en la subregión Sabanas se reportó un $80.7 \%$ en el grupo etario de 13 a 15 años, en comparación con el grupo de 10 a 12 años, con un 66.7\%; mientras que en la subregión Golfo de Morrosquillo los porcentajes para ambos rangos de edad fueron $75.6 \% \mathrm{y}$ $64.3 \%$,respectivamente.

Referente a la dimensión orden, se hallaron diferencias por grupos de edad $(\mathrm{p}<0.05)$ en el ítem soy organizado (a) con las responsabilidades diarias, predominando la categoría siempre en un $66.7 \%$ en el grupo etario de 10 a 12 años correspondiente a la subregión Golfo de Morrosquillo, mientras que en el grupo de 13 a 15 años alcanzó un $37.8 \%$; por otra parte, en la subregión Sabanas se obtuvo un $42.6 \%$ en estudiantes en el rango de 10 a 12 años en contraste con un $37.4 \%$ en adolescentes con edades de 13 a 15 años.

Respecto a estilos de vida por grado escolar e instituciones educativas por subregiones, los hallazgos reportan un estilo adecuado en los grados 6 y 9 de las instituciones ubicadas en la subregión Golfo de Morrosquillo, con los siguientes promedios: en el grado 6, nutrición (5.08); en el grado 9, actividad física y social (2.18), tipo de personalidad y satisfacción escolar (5.06) e imagen interior (3.29). Se destaca, que en la dimensión familiares y amigos, todos los grados de las dos subregiones alcanzaron un estilo de vida alto, con mayor promedio (4.75) en el grado 9, correspondiente a la subregión Golfo de Morrosquillo. En cuanto a la dimensión control de salud y sexualidad, los mayores promedios se percibieron en los estudiantes con estilo de vida adecuado de los grados 6 y 7 de la subregión Sabanas. En general, las medias y desviaciones estándar registraron diferencias muy significativas en las dimensiones familiares y orden ( $p$ valor 0.001 y 0.006 );respecto a la actividad física-social, nutrición, tipo de personalidad-satisfacción escolar, imagen interior, control de salud y sexualidad, se reportaron diferencias significativas $(\mathrm{p}<0.05)$.

En las dimensiones toxicidad, alcohol, sueño y estrés, no se reportaron diferencias significativas entre las medias de las puntuaciones ( $\mathrm{p}>0.05$ ).

En lo concerniente a la dimensión control de salud y sexualidad, en el ítem asisto a consulta para vigilar mi estado de salud,la mayoría de los estudiantes (60.8\%) de las tres instituciones educativas de la subregión Sabanas y la Institución $N^{\circ} 1$ del Golfo de Morrosquillo expresaron que algunas veces lo hacen; mientras que en el ítem converso con integrantes de mi familia sobre sexualidad, la mayoría de los estudiantes de las instituciones educativas de las dos subregiones consideraron que nunca lo hacen. 


\section{Discusión}

Entre los hallazgos referentes a las diferencias estadísticas muy significativas $(\mathrm{p}<0.01)$ relacionados con los estilos de vida y las variables género, edad e instituciones educativas por subregiones, se observa la influencia de lo sociocultural en el comportamiento de cada individuo o colectivo; por tanto, cada región al crear sus propios comportamientos, define su modo de vida con factores protectores o no para la salud(4), lo que contribuye a una mayor comprensión de comportamientos disímiles en los adolescentes. Al respecto, el Modelo de Promoción de la Salud de la teorista Nola Pender, sostiene que entre los factores que afectan la salud están los personales, como los biológicos, psicológicos y socioculturales, y dentro de los factores que la influencian, los situacionales o del contexto, que pueden impedir de manera directa o indirecta conductas saludables (21).Es por eso, que desde este enfoque los resultados de este estudio muestran que los factores biológicos como la edad, el género y la etapa de adolescencia,así como las variables relacionadas con la autoimagen interior, relaciones interpersonales y el mismo contexto sociocultural de cada subregión fueron determinantes en el nivel de estilo de vida reportado por los participantes.

Llama la atención,que la relación entre estilos de vida, grado escolar y subregión de procedencia, contrastan con los resultados obtenidos por los estudiantes de diversos grados, los que en su mayoría alcanzaron la categoría alto que correspondieron a los ubicados en losgrados 7 y 9 de la subregión Golfo de Morrosquillo; mientras que en los estudiantes de la subregión Sabanas predominó la categoría bajo en los de grado 7;situación que confirma la necesidad de estudiar las condiciones de vida de los adolescentes en diversos contextos, para favorecer de manera progresiva y sostenida la reducción a su exposición a factores de riesgo que pueden incidir en el modo y condiciones de vida (22).

En lo referente a los estilos de vida, edad y subregiones, se comprobó que a mayor edad (13 a 15 años)hay un estilo de vida adecuado en los adolescentes de las instituciones educativas de las dos subregiones,con respecto al grupo de menor rango (10-12 años), datos que contrastan con los hallazgos de Vélez y Betancurth(23), en donde los porcentajes más altos se ubicaron en el rango de menor edad (10 - 12 años); igualmente sostienen que a mayor edad se observa una variación del cambio en el estilo de vida hacia la zona de riesgo, situación que permite afirmar, que no necesariamente los estilos de vida con mayor riesgo se ubiquen en la etapa de adolescencia media. Desde esta perspectiva, es recomendable realizar intervenciones que fomenten prácticas saludables desde muy temprana edad, sustentado, en que las situaciones que afecten la salud en la infancia, pueden tener consecuencias en la adolescencia, y a su vez los problemas que ocurran en ésta, redundarán en posteriores etapas (5).

Hay que precisar, que desde los diversos contextos de donde proceden los adolescentes con características sociodemográficas distintas y apoyados en las actuales políticas de prevención de la ECNT, es posible abordar integralmente la problemática de los diversos comportamientos inadecuados para la salud, con estrategias preventivas de sensibilización hacia la adquisición de conocimientos, actitudes y prácticas positivas. Por ello, a la luz de los resultados del presente estudio, desde la perspectiva de la prevención de hábitos nocivos para la salud, implica intervenir en los participantes con estilos de vida bajo, según género e instituciones educativas, y por tanto trabajar la dimensión actividad física y social que reportó diferencias muy significativas (p valor 0.001 y 0.00 ); así como la dimensión tipo de personalidad y satisfacción escolar con diferencia significativa ( $\mathrm{p}$ valor 0.046 ).

Las evidencias anteriores permiten inferir que existe la necesidad de establecer alianzas estratégicas pactadas entre los sectores educativos y de salud, con el fin de avanzar de manera coherente hacia el logro de las metas planteadas en la Estrategia Mundial para la salud de la mujer y el adolescente (2016 - 2030), por relacionarse con los estilos de vida y la aparición de las ECNT(4).De igual forma, la OMS plantea acciones tales como, instituir vínculos estratégicos con los sistemas de salud, la cobertura sanitaria universal y la educación para alcanzar la salud,a través de la promoción de estilos de vida en los que se prevengan las enfermedades no transmisibles y sus factores de riesgo (24). En este sentido, la aplicación del Modelo de Promoción de la Salud de Nola Pender(21), puede aportar a la situación encontrada beneficios para la salud, dado que esté concibe la naturaleza multifacética de las personas en su interacción con el entorno; también, enfatiza en las características y experiencias individuales, así como en los conocimientos y afectos; este enfoque facilitará la adopción de comportamientos saludables en los participantes del estudio. 
ISSN-PRINT

1794-9831

E-ISSN 2322-7028

Vol. 17 No. 1

Ene - Abr 2020

Cúcuta, Colombia
Cabe resaltar, que los estilos de vida en las dimensiones familiares, amigos y orden, por grado cursado e instituciones educativas, registraron medias y desviaciones estándar con diferencias muy significativas ( $\mathrm{p}$ valor 0.001 y 0.006 ) respectivamente, cuyos resultados se ubicaron en las categorías de estilo alto y adecuado, lo que sugiere, que los participantes en su mayoría se encuentran en condiciones favorables en estos dominios. Es por eso que el ámbito escolar, además del familiar, se constituye en un espacio para la prevención de conductas de riesgo y la promoción de la salud, puesto que representa un contexto de aprendizaje e interacción donde se desarrolla el adolescente (25).

En cuanto a los datos sobre control de la salud y sexualidad, la mayoría de los adolescentes expresaron una ineficiente comunicación con los padres para abordar temas de sexualidad, resultados similares a los de Manrique et al. (26), quienes reportaron un riesgo alto de la conducta sexual en la comunicación parental, aspectos que en ambos estudios, posiblemente conlleve al adolescente a obtener información con personas externas al núcleo familiar.

Con respecto, a la dimensión que tiene que ver con la actividad física y social, se reporta que los estudiantes del género femenino realizan menos actividad física, datos que coinciden con los resultados de diversos estudios realizados en adolescentes escolarizados $(23,27,28)$. Cabe señalar que los lineamientos sobre la actividad fisica en la adolescencia resaltan la necesidad de realizar actividad física de intensidad moderada o vigorosa por los beneficios que genera en la salud(4). La actividad física se constituye en un elemento de importancia en la prevención de muchas enfermedades crónicas y mejora la calidad de vida del individuo (29).

Por otro lado, en lo referente a nutrición se encontró diferencias significativas $(\mathrm{p}<0.05)$ en los estudiantes por subregión. Los datos obtenidos muestran que los adolescentes de la subregión Golfo de Morrosquillo, solo el grado 6 alcanzó un estilo adecuado, mientras que en la subregión Sabanas predominó el estilo de vida bajo, representado en hábitos y consumo de una dieta inadecuada; resultados que difieren con los hallazgos de Gaither y Pimienta (30), quienes informaron que la mayoría de los estudiantes de secundaria (82\%) consumen una dieta balanceada. En este componente, la orientación alimentaria para los adolescentes de 10 a 17 años de edad, consiste en restringir la ingesta de sal a menos de $5 \mathrm{~g}$ por día, limitar el consumo de alimentos procesados y comidas rápidas, consumir cinco a siete raciones de frutas y verduras por día y limitar el consumo de grasas (4). Se debe mencionar que López et al. (31) sostienen, que el entorno escolar tiene una influencia determinante en la problemática de hábitos alimentarios poco saludables, además, destacan sobre la importancia de implementar una oferta alimentaria que permita inducir prácticas saludables en los escolares y adolescentes. En este contexto, es imprescindible que desde la infancia se fomenten conductas alimentarias saludables que permitan un óptimo crecimiento y desarrollo del adolescente; por tanto,es necesario fortalecer en los participantes del estudio y en su familia todo lo que tiene que ver con los hábitos alimentarios desde el contexto cultural y educativo de cada subregión.

En cuanto al tipo de personalidad y satisfacción escolar,en las cinco instituciones educativas se evidencia, que sólo los estudiantes del género masculino de la subregión Golfo de Morrosquillo alcanzaron un estilo de vida alto, en contraste con el resto de estudiantes que reportó un estilo bajo. En la dimensión imagen interior, se destaca el ítem me siento tenso(a) o estresado(a), condición que se presentó en mayor proporción en los estudiantes con edades entre 13 a 15 años, quienes expresaron que algunas veces lo han vivenciado. Lo expresado anteriormente por los estudiantes induce a explorar en el adolescente sus pensamientos y sentimientos en la interacción cotidiana, más aún cuando la tendencia es sustituir de forma progresiva al círculo familiar por grupos de amigos para aclarar inquietudes, situación que en ocasiones es causa de estrés en los adolescentes (32).

Lo anterior insta a implementar estrategias para fortalecer el crecimiento personal, el control de situaciones cotidianas y mejorar los estados de ánimo, si se tienen en cuenta los cambios físicos y psicosociales que experimenta el adolescente en esta etapa. En este sentido, Salavera y Usán(33) sostienen que el buen uso del humor puede proporcionar al individuo soporte emocional, servir en la gestión de conflictos y lograr satisfacción con la vida. Por otra parte, Uribe et al (34) afirman que los adolescentes que utilizan de forma recurrente estrategias de afrontamiento dirigidas a la resolución de problemas y búsqueda de apoyo social presentan mejores niveles de bienestar psicológico.

Finalmente, es fundamental impulsar alianzas estratégicas entre el sector educativo y salud, con el fin de 
ayudar de manera integral a la población adolescente, mediante acciones dirigidas a la promoción de estilos de vida saludables desde la infancia.

\section{Conclusiones}

Desde el contexto de las características sociodemográficas de los adolescentes de la básica secundaria de cinco instituciones educativas de las subregiones Golfo de Morrosquillo y Sabanas, se concluye, que la edad promedio fue de 13.1 años, con porcentajes similares por género $(50 \%)$, procedentes del municipio de Sincelejo y Santiago de Tolú, con predominio de los estratos socioeconómico 1 y 2 .

En las dos subregiones del departamento de Sucre, predominó un estilo de vida adecuado en los adolescentes con edades entre 13 y 15 años. Sin embargo, en la subregión del Golfo de Morrosquillo predominó la categoría de estilo de vida bajo en los estudiantes del género femenino.

La relación entre estilos de vida y las variables género, edad e instituciones educativas por subregión, se reportaron diferencias muy significativas, lo que evidenció la influencia de lo sociocultural en el comportamiento de hábitos de vida de los adolescentes

En los estudiantes de las dos subregiones se encontró que entre los estilos de vida y las variables género, edad e instituciones educativas, según las dimensiones estudiadas, diferencias muy significativas en las dimensiones actividad física y social, sueño y estrés, nutrición, familiares y amigos. También hay diferencias significativas en el tipo de personalidad y la satisfacción escolar, imagen interior y orden. Estos hallazgos conducen a impulsar en esta población,investigaciones de intervención dirigidas al fomento de comportamientos saludables desde su contexto sociocultural, para fortalecer los factores protectores y controlar el riesgo de padecer enfermedades crónicas no transmisibles.

Es pertinente el compromiso del profesional de la enfermería en la promoción de la salud a partir del estado de salud en la población adolescente, con el fin de establecer planes de acción que conlleven a asumir conductas saludables acordes con las características de cada contexto, para así alcanzar un mayor impacto en la consecución de mejores oportunidades y estilos de vida.

Los resultados del estudio representan para la enfermería nuevos retos en el cuidado de la población adolescente, orientados a la aplicación de modelos propios con énfasis en la promoción de estilos de vida saludables y el fortalecimiento del autocuidado, promoviendo la participación activa en la planeación y gestión de políticas públicas que permitan la articulación efectiva entre el sector salud y la academia, para lograr así una mejor atención integral al adolescente.

\section{Conflicto de Intereses}

Las autoras declaran no tener ningún conflicto de intereses

\section{Referencias Bibliográficas}

1. Organización Mundial de la Salud. Desarrollo en la adolescencia [Internet]. [consultado 2019 Mar 4]. Disponible en: https://www.who.int/maternal_child_adolescent/topics/adolescence/dev/es/.

2. Republica de Colombia. Ministerio de Salud y Protección Social. Plan Decenal de Salud Pública 2012 - 2021. Dimensión vida saludable y condiciones no trasmisibles [Internet]. Santa Fé de Bogotá; 2013. Disponible en: https://www.minsalud.gov.co/plandecenal/Documents/dimensiones/dimensionvidasaludable-condicionesno-transmisibles.pdf.

3. Organización Mundial de la Salud. Salud para los adolescentes del mundo. Una segunda oportunidad en la segunda década: resumen [Internet]. Ginebra, Suiza; 2014 [consultado 2019 Mar 4]. Disponible en: https://apps.who.int/iris/bitstream/handle/10665/141455/WHO_FWC_MCA_14.05_spa. pdf? sequence $=1 \&$ isAllowed $=\mathrm{y}$.

4. Organizacion Panamericana de la Salud. Aceleración mundial de las medidas para promover la salud de los adolescentes. (Guía AA-HAI): Orientación para la aplicación en los países [Internet]. Washington, DC, Estados Unidos; 2018 [consultado 2019 Mar 6]. Disponible en: http://iris.paho.org/xmlui/ handle/123456789/49181. 
ISSN-PRINT

$1794-9831$

E-ISSN 2322-7028

Vol. 17 No. 1

Ene - Abr 2020

Cúcuta, Colombia
5. Johnson MC, Thomas AL, Greenlief CM. Impact of Frozen Storage on the Anthocyanin and Polyphenol Contents of American Elderberry Fruit Juice. J Agric Food Chem [Internet]. 2015 [consultado 2019 Mar 9];63(23):5653-9. Disponible en: http://scielo.sld.cu/scielo.php?script=sci arttext\&pid=S1727897X2017000100011. DOI:10.1021/acs.jafc.5b01702

6. Municipio Santiago de Tolú. Departamento de Sucre. Colombia. Plan de Desarrollo Territorial. Municipio de Santiago de Tolú. El plan de todos por el cambio. 2016 - 2019 [Internet]. Santiago de Tolú; 2016. Disponible en: http://www.santiagodetolu-sucre.gov.co/Transparencia/PlaneacionGestionyControl/Plan de Desarrollo Territorial 2016-2019.pdf.

7. Municipio de Sincelejo. Departamento de Sucre. Colombia. Plan de Desarrollo 2016 - 2019. Sincelejo, Ciudad con Visión [Internet]. Sincelejo; 2016. Disponible en: http://www.alcaldiadesincelejo. gov.co/Ciudadanos/ProyectosNormatividad/Plan de Desarrollo Municipal 2016 - 2019.pdf.

8. World Health Organization. Global Health Estimates 2016: Disease burden by Cause, Age, Sex, by Country and by Region, 2000-2016 [Internet]. 2018 [consultado 2019 Mar 9]. Disponible en: https:// www.who.int/healthinfo/global_burden_disease/estimates/en/index1.html.

9. World Health Organization. Global Status Report on noncommunicable diseases 2014 [Internet]. Geneva, Switzerland; 2014 [consultado 2019 Mar 9]. Disponible en: https://apps.who.int/iris/bitstream/ handle/10665/148114/9789241564854_eng.pdf?sequence $=1$.

10. Organización Mundial de la Salud. Salud de la madre, el recién nacido, del niño y del adolescente. [Internet]. Consultado[2019 Nov 28]disponible En: https://www.unicef.org/argentina/media/1536/file/ Suicidio.pdf

11. Organización Mundial de la Salud. Prevención del suicidio. [Internet]. Consultado[2019 Nov 28]disponible En:_https://www.who.int/mental_health/prevention/suicide/suicideprevent/es/

12. World Health Organization. Global action plan on physical activity 2018-2030: more active people for a healthier world [Internet]. Geneva, Switzerland; 2018 [consultado 2019 Mar 12]. Disponible en: https://apps.who.int/iris/bitstream/handle/10665/272722/9789241514187-eng.pdf.

13. Aragonés Clemente MT, Fernández Navarro P, Ley Vega de Seoane V. Actividad física y prevalencia de patologías en la población española [Internet]. Madrid, España: Ministerio de Educación, Cultura y Deporte; 2016 [consultado 2019 Apr 18]. Disponible en: https://sede.educacion.gob.es/ publiventa/d/21268/19/1.

14. Organización Mundial de la Salud. Conferencia de las Partes en el Convenio Marco de la OMS para el Control del Tabaco. Sexta Reunión. Sistemas electrónicos de administración de nicotina: Informe de la OMS [Internet]. Moscú, Rusia; 2014. Disponible en: http://apps.who.int/gb/fctc/PDF/cop6/FCTC COP6 10Rev1-sp.pdf.

15. Observatorio de Drogas de Colombia. Estudio Nacional de Consumo de Sustancias Psicoactivas en Población Escolar Colombia - 2016. Consultado [2019 Nov 28]. Disponible En : http://www.odc.gov. co/Portals/1/publicaciones/pdf/consumo/estudios/nacionales/CO03142016 estudio consumo escolares 2016.pdf

16. Ministerio de Salud y Protección Social. Análisis de Situación de Salud (ASIS) Colombia, V Edición. 2018. Disponible: https:/www.minsalud.gov.co/sites/rid/Lists/BibliotecaDigital/RIDE/VS/ED/PSP/ asis-colombia-2018.pdf PAG 186

17. Departamento de Sucre. Colombia. Plan Departamental de Desarrollo 2016 - 2019. Sucre Progresa en Paz [Internet]. 2016. Disponible en: https://sucre.micolombiadigital.gov.co/sites/sucre/content/ files/000023/1140 plan-departamental-de-desarrollo-20162019.pdf.

18. De Arco-Canoles OdelC, Suarez-Calle ZK. Rol de los profesionales de enfermería en el sistema de salud colombiano. Univ. Salud. 2018;20(2):171-182. DOI: http://dx.doi.org/10.22267/rus.182002.121

19. Betancurth Loaiza DP, Vélez Álvarez C, Jurado Vargas L. Validación de contenido y adaptación del cuestionario Fantastico por técnica Delphi. Salud Uninorte. 2015;31(2):214-27. 
20. República de Colombia. Ministerio de Salud. Resolución No008430 de 1993, octubre 4, por la cual se establecen las normas científicas, técnicas y administrativas para la investigación en salud [Internet]. Santa Fé de Bogotá; 1993. Disponible en: https://www.minsalud.gov.co/sites/rid/lists/bibliotecadigital/ride/de/dij/resolucion-8430-de-1993.pdf.

21. Sakraida TJ, Wilson J. Modelo de promoción de la Salud. Nola J. Pender. In: Raile Alligood M, editor. Modelos y teorías en enfermería. 9a edición. Barcelona, España: Elsevier; 2018. p. 323-38.

22. Pedersen BK, Saltin B. Exercise as medicine - evidence for prescribing exercise as therapy in 26 different chronic diseases. Scand J Med Sci Sports [Internet]. 2015;25(S3):1-72. Disponible en: http:// doi.wiley.com/10.1111/sms.12581. DOI:10.1111/sms.12581

23. Vélez C, Betancurth P. Estilos de vida en adolescentes escolarizados de un municipio colombiano. Rev Cubana Pediatr. 2015;87(4):440-8.

24. Organización Mundial de la Salud. Es hora de actuar: informe de la Comisión independiente de alto nivel de la OMS sobre enfermedades no transmisibles [Internet]. Ginebra, Suiza; 2018. Disponible en: http://apps.who.int/iris/bitstream/handle/10665/272712/9789243514161-spa.pdf?ua=.

25. Vallejo Alviter NG, Martínez Moctezuma E. Impacto de la promoción de estilos de vida saludable para mejorar la calidad de vida. Drugs Addict Behav [Internet]. 2017 [consultado 2019 Apr 18];2(2):225-35. Disponible en: http://www.funlam.edu.co/revistas/index.php/DAB/article/ view/2442. DOI: $10.21501 / 24631779.2442$

26. Manrique de Lara Suáre D, Figueroa Sánchez I, Miguel Carrasco Muñoz M, Cisneros Martínez L. Conflicto interparental desde la perspectiva de los hijos y conductas sexuales de riesgo en adolescentes en una Institución Educativa de las Moras, Huánuco. Investig Vald [Internet]. 2018;12(3):13746. Disponible en: http://revistas.unheval.edu.pe/index.php/riv/article/view/148. DOI:10.33554/ riv.12.3.148

27. Medina C, Jáuregui A, Campos-Nonato I, Barquera S. Prevalencia y tendencias de actividad física en niños y adolescentes: resultados de Ensanut 2012 y Ensanut MC 2016. Salud Publica Mex [Internet]. 2018;60(3, may-jun):263. Disponible en: http://www.saludpublica.mx/index.php/spm/article/ view/8819. DOI:10.21149/8819

28. Ibarra Mora JL, Ventura Vall-Llovera C, Hernández-Mosqueira C. Hábitos de vida saludable de actividad física, alimentación, sueño y consumo de tabaco y alcohol, en estudiantes adolescentes chilenos. Sport Sci J Sch Sport Phys Educ Psychomot [Internet]. 2019 [consultado 2019 May 3];5(1):7084. Disponible en: http://revistas.udc.es/index.php/SPORTIS/article/view/sportis.2019.5.1.3500. DOI:10.17979/sportis.2019.5.1.3500

29. Córdoba García R, Camarelles Guillem F, Muñoz Seco E, Gómez Puente JM, Ramírez Manent JI, San José Arango J, et al. Recomendaciones sobre el estilo de vida. Atención Primaria [Internet]. 2016;48(S1):27-38. Disponible en: http://linkinghub.elsevier.com/retrieve/pii/S021265671630186X. DOI:10.1016/S0212-6567(16)30186-X

30. Gaither Jiménez LE, Pimienta Prieto JH. Descripción de los estilos de vida y Factores de riesgo en niños y adolescentes de Tamaulipas [Internet]. Revista panamericana de pedagogía, saberes y quehaceres del pedagogo. Universidad Panamericana; 2017 [consultado 2019 May 3]. 171-191 p. Disponible en: https://biblat.unam.mx/en/revista/revista-panamericana-de-pedagogia-saberes-y-quehaceres-delpedagogo/articulo/descripcion-de-los-estilos-de-vida-y-factores-de-riesgo-en-ninos-y-adolescentesde-tamaulipas.

31. López Daza GA, Torres P K, Gómez García CF. La alimentación escolar en las instituciones educativas públicas de Colombia. Análisis normativo y de la política pública alimentaria. Prolegómenos [Internet]. 2017 [consultado 2019 May 3];20(40):97-112. Disponible en: https://revistas.unimilitar. edu.co/index.php/dere/article/view/3043. DOI:10.18359/prole.3043

32. Ramos-Vidal I. Popularidad y relaciones entre iguales en el aula: un estudio prospectivo. Psicol Educ 
ISSN-PRINT

$1794-9831$

E-ISSN 2322-7028

Vol. 17 No. 1

Ene - Abr 2020

Cúcuta, Colombia

[Internet]. 2016;22(2):113-24. Disponible en: http://journals.copmadrid.org/psed/articulo.php?id=8c0 1a75941549a705cf7275e41b21f0d. DOI:10.1016/j.pse.2015.12.001

33. Salavera C, Usán P. Relación entre los estilos del humor y la satisfacción con la vida en estudiantes de Secundaria. Eur J Investig Heal Psychol Educ [Internet]. 2018 [consultado 2019 May 2];7(2):8797. Disponible en: https://formacionasunivep.com/ejihpe/index.php/journal/article/view/198. DOI:10.30552/ejihpe.v7i2.198

34. Uribe Urzola A, Ramos Vidal I, Villamil Benítez I, Palacio Sañudo JE. La importancia de las estrategias de afrontamiento en el bienestar psicológico en una muestra escolarizada de adolescentes. PSICOGENTE [Internet]. 2018 [consultado 2019 May 2];21(40):440-57. Disponible en: http://revistas. unisimon.edu.co/index.php/psicogente/article/view/3082. DOI:10.17081/psico.21.40.3082 